\title{
Harmonic Aspects in an $\eta$-Ricci Soliton
}

\author{
Adara M. Blaga \\ (Communicated by Uday Chand De)
}

\begin{abstract}
We characterize $\eta$-Ricci solitons $(g, \xi, \lambda, \mu)$ in some special cases when the 1 -form $\eta$, which is the $g$-dual of $\xi$, is a harmonic or a Schrödinger-Ricci harmonic form. We also provide necessary and sufficient conditions for $\eta$ to be a solution of the Schrödinger-Ricci equation and point out the relation between the three notions in our context. In particular, we apply these results to a perfect fluid spacetime and using Bochner-Weitzenböck techniques, we formulate some more conclusions for gradient solitons and deduce topological properties of the manifold and its universal covering.
\end{abstract}

Keywords: gradient Ricci solitons; Schrödinger-Ricci equation; harmonic form.

AMS Subject Classification (2020): Primary: 35C08; 53C25.

\section{Introduction}

Self-similar solutions to the Ricci flow, the Ricci solitons [31] have been studied in different geometrical contexts on complex, contact and paracontact manifolds. The more general notion of $\eta$-Ricci soliton was introduced by J. T. Cho and M. Kimura [22] on real hypersurfaces in a Kähler manifold and treated in complex space forms [21], Euclidean hypersurfaces [1], paracontact geometries [4], [5], [17], [18], [19], [26]. Different geometrical aspects of Ricci and $\eta$-Ricci solitons have been studied by author in [6], [13], [15]. Further generalizations of this notion and properties of other geometrical solitons can be found in [9], [11] and [2], [14].

A particular case of solitons arise when they evolve by diffeomorphism generated by a gradient vector field, namely when the potential vector field is the gradient of a smooth function. The gradient vector fields play a central rôle in Morse-Smale theory [37] and some aspects of gradient $\eta$-Ricci solitons were discusses by author in [3], [7], [8], [10], [12], [16].

In Section 2, after we point out the basic properties of an $\eta$-Ricci soliton $(g, \xi, \lambda, \mu)$, we provide necessary and sufficient conditions for the $g$-dual 1-form of the potential vector field $\xi$ to be a solution of the SchrödingerRicci equation, a harmonic or a Schrödinger-Ricci harmonic form and characterize the 1-forms orthogonal to $\eta$. We end these considerations by discussing the case of a perfect fluid spacetime. In Section 3 we formulate the results for the special case of gradient solitons and deduce topological properties of the manifold and its universal covering [33].

\section{Geometrical aspects of $\eta$}

Let $(M, g)$ be an $n$-dimensional Riemannian manifold, $n>2$, and denote by $b: T M \rightarrow T^{*} M, b(X):=i_{X} g$, $\sharp: T^{*} M \rightarrow T M, \sharp:=b^{-1}$ the musical isomorphism. Consider the set $\mathcal{T}_{2, s}^{0}(M)$ of symmetric $(0,2)$-tensor fields on $M$ and for $Z \in \mathcal{T}_{2, s}^{0}(M)$, denote by $Z^{\sharp}: T M \rightarrow T M$ and by $Z_{\sharp}: T^{*} M \rightarrow T^{*} M$ the maps defined as follows:

$$
g\left(Z^{\sharp}(X), Y\right):=Z(X, Y), Z_{\sharp}(\alpha)(X):=Z(\sharp(\alpha), X) .
$$

We also denote by $Z^{\sharp}$ the map $Z^{\sharp}: T^{*} M \times T^{*} M \rightarrow C^{\infty}(M)$ :

$$
Z^{\sharp}(\alpha, \beta):=Z(\sharp(\alpha), \sharp(\beta))
$$


and can identify $Z_{\sharp}$ with the map also denoted by $Z_{\sharp}: T^{*} M \times T M \rightarrow C^{\infty}(M)$ :

$$
Z_{\sharp}(\alpha, X):=Z_{\sharp}(\alpha)(X) .
$$

Given a vector field $X$, its $g$-dual 1-form $X^{b}=: b(X)$ is said to be a solution of the Schrödinger-Ricci equation if it satisfies:

$$
\operatorname{div}\left(L_{X} g\right)=0,
$$

where $L_{X} g$ denotes the Lie derivative along the vector field $X$.

It is known that [24]:

$$
\operatorname{div}\left(L_{X} g\right)=\left(\Delta+S_{\sharp}\right)\left(X^{b}\right)+d(\operatorname{div}(X)),
$$

where $\Delta$ denotes the Laplace-Hodge operator on forms w.r.t. the metric $g$ and $S$ the Ricci curvature tensor field. Denoting by $Q$ the Ricci operator defined by $g(Q X, Y):=S(X, Y)$, for any vector fields $X$ and $Y$, by a direct computation we deduce that $S_{\sharp}(\gamma)=i_{Q \gamma^{\sharp}} g$, for any 1-form $\gamma$.

$\eta$-Ricci solitons. We are interested to find the necessary and sufficient conditions for the $g$-dual 1-form $\eta$ of the potential vector field $\xi$ in an $\eta$-Ricci soliton to be a solution of the Schrödinger-Ricci equation, a harmonic or Schrödinger-Ricci harmonic form.

Consider the equation:

$$
L_{\xi} g+2 S+2 \lambda g+2 \mu \eta \otimes \eta=0,
$$

where $g$ is a Riemannian metric, $S$ its Ricci curvature tensor field, $\xi$ a vector field, $\eta$ a 1-form and $\lambda$ and $\mu$ are real constants. The data $(g, \xi, \lambda, \mu)$ which satisfy the equation (2.3) is said to be an $\eta$-Ricci soliton on $M$ [22]; in particular, if $\mu=0,(g, \xi, \lambda)$ is a Ricci soliton [31] and it is called shrinking, steady or expanding according as $\lambda$ is negative, zero or positive, respectively [25]. If the potential vector field $\xi$ is of gradient-type, $\xi=\operatorname{grad}(f)$, for $f$ a smooth function on $M$, then $(g, \xi, \lambda, \mu)$ is called a gradient $\eta$-Ricci soliton.

Taking the trace of the equation (2.3) we obtain:

$$
\operatorname{div}(\xi)+s c a l+\lambda n+\mu|\xi|^{2}=0 .
$$

From a direct computation we get:

$$
\operatorname{div}(\eta \otimes \eta)=\operatorname{div}(\xi) \eta+\nabla_{\xi} \eta
$$

Now taking the divergence of (2.3) and using (2.2) we obtain:

$$
\operatorname{div}\left(L_{\xi} g\right)+d(s c a l)+2 \mu\left[\operatorname{div}(\xi) \eta+\nabla_{\xi} \eta\right]=0 .
$$

Schrödinger-Ricci solutions. We say that a 1-form $\gamma$ is a solution of the Schrödinger-Ricci equation if

$$
\left(\Delta+S_{\sharp}\right)(\gamma)+d\left(\operatorname{div}\left(\gamma^{\sharp}\right)\right)=0 .
$$

Theorem 2.1. Let $(g, \xi, \lambda, \mu)$ be an $\eta$-Ricci soliton on the $n$-dimensional manifold $M$ with $\eta$ the $g$-dual of $\xi$. Then $\eta$ is a solution of the Schrödinger-Ricci equation if and only if

$$
d(\text { scal })=2 \mu\left[\left(\text { scal }+\lambda n+\mu|\xi|^{2}\right) \eta-\nabla_{\xi} \eta\right] .
$$

Moreover, in this case, scal is constant if and only if $\mu=0$ (which yields a Ricci soliton) or $\left(\right.$ scal $\left.+\lambda n+\mu|\xi|^{2}\right) \eta=$ $\nabla_{\xi} \eta$.

Proof. From (2.3), (2.4), (2.5) and

$$
2 \operatorname{div}(S)=d(\text { scal })
$$

it follows that $\eta$ is a solution of the Schrödinger-Ricci equation if and only if (2.7) holds.

Remark 2.1. Under the hypotheses of Theorem 2.1, if the potential vector field is of constant length $k$, then from (2.7) we deduce that the scalar curvature is constant if either the soliton is a Ricci soliton or, $\left(s c a l+\lambda n+\mu k^{2}\right) \eta=$ $\nabla_{\xi} \eta$ which implies $s c a l=-\lambda n-\mu k^{2}$. 
Corollary 2.1. Let $(g, \xi, \lambda, \mu)$ be an $\eta$-Ricci soliton on the $n$-dimensional manifold $M$ with $\eta$ the $g$-dual of $\xi$ and assume that $\eta$ is a nontrivial solution of the Schrödinger-Ricci equation. If scal is constant and $\mu \neq 0$, then $\frac{1}{2|\xi|^{2}} \xi\left(|\xi|^{2}\right)-\mu|\xi|^{2}=$ scal $+\lambda n$ (constant).

Proof. Under the hypotheses, from (2.7) we obtain:

$$
\left(s c a l+\lambda n+\mu|\xi|^{2}\right) \eta=\nabla_{\xi} \eta,
$$

applying $\xi$ and taking into account that

$$
\left(\nabla_{\xi} \eta\right) \xi=\frac{1}{2} \xi\left(|\xi|^{2}\right)
$$

we deduce that $\left(\right.$ scal $\left.+\lambda n+\mu|\xi|^{2}\right)|\xi|^{2}=\frac{1}{2} \xi\left(|\xi|^{2}\right)$.

For the case of Ricci solitons, from Theorem 2.1 we have:

Corollary 2.2. If $(g, \xi, \lambda)$ is a Ricci soliton on the $n$-dimensional manifold $M$ and $\eta$ is the $g$-dual of $\xi$, then $\eta$ is a solution of the Schrödinger-Ricci equation if and only if the scalar curvature of the manifold is constant.

Schrödinger-Ricci harmonic forms. We say that a 1-form $\gamma$ is Schrödinger-Ricci harmonic if

$$
\left(\Delta+S_{\sharp}\right)(\gamma)=0 .
$$

From (2.6), (2.4) and (2.5) we deduce:

Theorem 2.2. Let $(g, \xi, \lambda, \mu)$ be an $\eta$-Ricci soliton on the $n$-dimensional manifold $M$ with $\eta$ the $g$-dual of $\xi$. Then $\eta$ is a Schrödinger-Ricci harmonic form if and only if $\mu=0$ (which yields a Ricci soliton) or

$$
\left(s c a l+\lambda n+\mu|\xi|^{2}\right) \eta=\nabla_{\xi} \eta-\frac{1}{2} d\left(|\xi|^{2}\right) .
$$

Remark 2.2. Under the hypotheses of Theorem 2.2, if $\mu \neq 0$, then from (2.8) we deduce that the scalar curvature is constant if and only if the potential vector field is of constant length.

Harmonic forms. We know that on a Riemannian manifold $(M, g)$, a 1-form $\gamma$ is harmonic (i.e. $\Delta(\gamma)=0)$ if and only if it is closed and divergence free.

Remark that on an $\eta$-Ricci soliton, a harmonic 1-form $\gamma$ is Schrödinger-Ricci harmonic if and only if

$$
\gamma \circ \nabla \xi+\lambda \gamma+\mu \gamma(\xi) \eta=0
$$

which implies (using the fact that $\left(\nabla_{X} \gamma\right)^{\sharp}=\nabla_{X} \gamma^{\sharp}$, for any vector field $X$ and any 1-form $\gamma$ ):

$$
\gamma^{\sharp} \in \operatorname{ker}\left[\nabla_{\xi} \eta+\left(\lambda+\mu|\xi|^{2}\right) \eta\right] .
$$

From (2.2) and (2.5) we deduce:

Theorem 2.3. Let $(g, \xi, \lambda, \mu)$ be an $\eta$-Ricci soliton on the $n$-dimensional manifold $M$ with $\eta$ the $g$-dual of $\xi$. Then $\eta$ is a harmonic form if and only if

$$
i_{Q \xi} g=\mu\left\{2\left[\left(s c a l+\lambda n+\mu|\xi|^{2}\right) \eta-\nabla_{\xi} \eta\right]+d\left(|\xi|^{2}\right)\right\} .
$$

For the case of Ricci solitons, from Theorem 2.3 we have:

Corollary 2.3. If $(g, \xi, \lambda)$ is a Ricci soliton on the $n$-dimensional manifold $M$ and $\eta$ is the $g$-dual of $\xi$, then $\eta$ is a harmonic form if and only if $\xi \in \operatorname{ker} Q$.

From (2.4), (2.8) and (2.9) we deduce:

Corollary 2.4. Let $(g, \xi, \lambda, \mu)$ be an $\eta$-Ricci soliton on the $n$-dimensional manifold $M$ with $\eta$ the $g$-dual of $\xi$. If $\eta$ is a harmonic form, then i) $\xi \in \operatorname{ker} Q$ and ii) the scalar curvature is constant if and only if the potential vector field $\xi$ is of constant length.

The relation between the cases when $\eta$ is a solution of the Schrödinger-Ricci equation, harmonic or the Schrödinger-Ricci harmonic form is stated in the following result: 
Lemma 2.1. Let $(g, \xi, \lambda, \mu)$ be an $\eta$-Ricci soliton on the $n$-dimensional manifold $M$ with $\eta$ the $g$-dual of $\xi$.

i) If $\eta$ is a solution of the Schrödinger-Ricci equation, then $\eta$ is:

a) Schrödinger-Ricci harmonic form if and only if scal $+\mu|\xi|^{2}$ is constant;

b) harmonic form if and only if $i_{Q \xi} g=d\left(\right.$ scal $\left.+\mu|\xi|^{2}\right)$; also $\eta$ harmonic implies $\xi \in \operatorname{ker} Q$.

ii) If $\eta$ is Schrödinger-Ricci harmonic form, then $\eta$ is:

a) a solution of the Schrödinger-Ricci equation if and only if scal $+\mu|\xi|^{2}$ is constant;

b) harmonic form if and only if $\xi \in \operatorname{ker} Q$.

iii) If $\eta$ is a harmonic form, then $\eta$ is:

a) a solution of the Schrödinger-Ricci equation if and only if $\xi \in \operatorname{ker} Q$;

b) Schrödinger-Ricci harmonic form if and only if $\xi \in \operatorname{ker} Q$.

We can synthesize:

i) if $s c a l+\mu|\xi|^{2}$ is constant, then $\eta$ is Schrödinger-Ricci harmonic if and only if it is a solution of the Schrödinger-Ricci equation;

ii) if $\xi \in \operatorname{ker} Q$, then $\eta$ is Schrödinger-Ricci harmonic if and only if it is harmonic.

1-forms orthogonal to $\eta$. We say that two 1 -forms $\gamma_{1}$ and $\gamma_{2}$ are orthogonal if $g\left(\gamma_{1}^{\sharp}, \gamma_{2}^{\sharp}\right)=0$ (i.e. $\left\langle\gamma_{1}, \gamma_{2}\right\rangle=0$, where $\left\langle\gamma_{1}, \gamma_{2}\right\rangle:=\sum_{i=1}^{n} \gamma_{1}\left(E_{i}\right) \gamma_{2}\left(E_{i}\right)$, for $\left\{E_{i}\right\}_{1 \leq i \leq n}$ a local orthonormal frame field).

Remark that $\gamma_{1}$ and $\gamma_{2}$ are orthogonal if and only if

$$
\gamma_{1}^{\sharp} \in \operatorname{ker} \gamma_{2} \text { or } \gamma_{2}^{\sharp} \in \operatorname{ker} \gamma_{1} .
$$

Theorem 2.4. Let $(g, \xi, \lambda, \mu)$ be an $\eta$-Ricci soliton on the $n$-dimensional manifold $M$ with $\eta$ the $g$-dual of $\xi$ and $\mu \neq 0$. If $\gamma$ is a 1-form, then $\gamma$ is orthogonal to $\eta$ if and only if

$$
\nabla_{\gamma^{\sharp}} \xi+Q \gamma^{\sharp}+\lambda \gamma^{\sharp} \in \operatorname{ker} \gamma .
$$

Proof. Observe that computing the soliton equation in $\left(\gamma^{\sharp}, \gamma^{\sharp}\right)$ and using the orthogonality condition we obtain:

$$
g\left(\nabla_{\gamma^{\sharp}} \xi, \gamma^{\sharp}\right)+g\left(Q \gamma^{\sharp}, \gamma^{\sharp}\right)+\lambda\left|\gamma^{\sharp}\right|^{2}=0
$$

which is equivalent to the condition (2.10).

Example We end these considerations by discussing the case of a perfect fluid spacetime $(M, g, \xi)[12]$. If we denote by $p$ the isotropic pressure, $\sigma$ the energy-density, $\lambda$ the cosmological constant, $k$ the gravitational constant, $S$ the Ricci curvature tensor field and scal the scalar curvature of $g$, then [12]:

$$
S=-\left(\lambda-\frac{s c a l}{2}-k p\right) g+k(\sigma+p) \eta \otimes \eta
$$

and the scalar curvature of $M$ is:

$$
s c a l=4 \lambda+k(\sigma-3 p) .
$$

From Theorem 2.1, we deduce that if $(g, \xi, a, b)$ is an $\eta$-Ricci soliton on $(M, g, \xi)$, then $\eta$ is a solution of the Schrödinger-Ricci equation if and only if

$$
k d(\sigma-3 p)=2 b\left\{[4(a+\lambda)-b+k(\sigma-3 p)] \eta-\nabla_{\xi} \eta\right\} .
$$

Moreover, the fluid is a radiation fluid (i.e. $\sigma=3 p$ ) if and only if $b=0$ (which yields the Ricci soliton) or $[4(a+\lambda)-b] \eta=\nabla_{\xi} \eta$ which implies $b=4(a+\lambda)$.

From Theorem 2.2, we deduce that if $(g, \xi, a, b)$ is an $\eta$-Ricci soliton on $(M, g, \xi)$, then $\eta$ is a Schrödinger-Ricci harmonic form if and only if $b=0$ (which yields a Ricci soliton) or

$$
[4(a+\lambda)-b+k(\sigma-3 p)] \eta=\nabla_{\xi} \eta
$$

which implies $b=4(a+\lambda)+k(\sigma-3 p)$.

From Theorem 2.3, we deduce that if $(g, \xi, a, b)$ is an $\eta$-Ricci soliton on $(M, g, \xi)$, then $\eta$ is a harmonic form if and only if

$$
\{4 b[4(a+\lambda)-b+k(\sigma-3 p)]-2 \lambda+k(\sigma+3 p)\} \eta=4 b \nabla_{\xi} \eta .
$$

For the case of Ricci soliton $(g, \xi, a)$ in a radiation fluid we obtain the constant pressure $p=\frac{\lambda}{3 k}$. 


\section{Applications to gradient solitons}

Let $f \in C^{\infty}(M), \xi:=\operatorname{grad}(f), \eta:=\xi^{b}$ and $\lambda$ and $\mu$ real constants. Then $\eta=d f$ and

$$
g\left(\nabla_{X} \xi, Y\right)=g\left(\nabla_{Y} \xi, X\right),
$$

for any $X, Y \in \mathfrak{X}(M)$. Also [5]:

$$
\begin{gathered}
\operatorname{trace}(\eta \otimes \eta)=|\xi|^{2}, \\
\operatorname{div}(\eta \otimes \eta)=\operatorname{div}(\xi) \eta+\frac{1}{2} d\left(|\xi|^{2}\right)
\end{gathered}
$$

and

$$
\nabla_{\xi} \eta=\frac{1}{2} d\left(|\xi|^{2}\right)
$$

For the gradient $\eta$-Ricci solitons we have:

Proposition 3.1. If $(g, \xi:=\operatorname{grad}(f), \lambda, \mu)$ is a gradient $\eta$-Ricci soliton on the $n$-dimensional manifold $M$ and $\eta=d f$ is the $g$-dual of $\xi$, then $\eta$ is a solution of the Schrödinger-Ricci equation if and only if

$$
d(\text { scal })=2 \mu\left[\left(\text { scal }+\lambda n+\mu|\xi|^{2}\right) d f-\frac{1}{2} d\left(|\xi|^{2}\right)\right] .
$$

Moreover, in this case, scal is constant if and only if $\mu=0$ (which yields a gradient Ricci soliton) or (scal $+\lambda n+$ $\left.\mu|\xi|^{2}\right) d f=\frac{1}{2} d\left(|\xi|^{2}\right)$.

Remark 3.1. Under the hypotheses of Proposition 3.1, if the potential vector field is of constant length $k$, then (3.5) becomes:

$$
d(s c a l)=2 \mu\left(s c a l+\lambda n+\mu k^{2}\right) d f,
$$

so the scalar curvature is constant if either the soliton is a gradient Ricci soliton or scal $=-\lambda n-\mu k^{2}$.

Remark 3.2. i) Taking into account that for a gradient vector field $\xi$ [10]:

$$
\operatorname{div}\left(L_{\xi} g\right)=2 d(\operatorname{div}(\xi))+2 i_{Q \xi} g,
$$

the condition for the $g$-dual $\eta=d f$ of the potential vector field $\xi:=\operatorname{grad}(f)$ of a gradient $\eta$-Ricci soliton $(g, \xi, \lambda, \mu)$ to be a solution of the Schrödinger-Ricci equation is:

$$
d\left(s c a l+\mu|\xi|^{2}\right)=i_{Q \xi} g .
$$

In this case, scal $+\mu|\xi|^{2}$ is constant if and only if $\xi \in \operatorname{ker} Q$ and from the $\eta$-Ricci soliton equation we obtain $\nabla_{\xi} \xi=-\left(\lambda+\mu|\xi|^{2}\right) \xi$. Applying $\eta$ we get $\lambda+\mu|\xi|^{2}=-\frac{1}{2|\xi|^{2}} \xi\left(|\xi|^{2}\right)$, therefore, if the length of $\xi$ is constant (also, the scalar curvature will be constant), then $|\xi|^{2}=-\frac{\lambda}{\mu}$, hence $\xi$ is a geodesic vector field.

ii) If $\xi$ is an eigenvector of $Q$ (i.e. $Q \xi=a \xi$, with $a$ a smooth function), then $\eta$ is a solution of the SchrödingerRicci equation if and only if $s c a l+\mu|\xi|^{2}-a f$ is constant. In particular, if $\xi \in \operatorname{ker} Q$, then $\eta$ is a solution of the Schrödinger-Ricci equation if and only if $\eta$ is a harmonic form.

iii) If $\eta$ is a Schrödinger-Ricci harmonic form, then $d\left(s c a l+\mu|\xi|^{2}\right)=2 i_{Q \xi} g$. In this case, scal $+\mu|\xi|^{2}$ is constant if and only if $\xi \in \operatorname{ker} Q$ and using the same arguments as in i) we deduce that $\xi$ is a geodesic vector field.

Also, an exact 1-form $d f$ is harmonic if and only if the function $f$ is harmonic. In the case of a gradient $\eta$-Ricci soliton, for $\eta$ harmonic form, denoting by $\Delta_{f}:=\Delta-\nabla_{\operatorname{grad}(f)}$ the $f$-Laplace-Hodge operator, the result stated in Theorem 3.2 from [10] becomes:

Theorem 3.1. Let $(g, \xi:=\operatorname{grad}(f), \lambda, \mu)$ be a gradient $\eta$-Ricci soliton on the $n$-dimensional manifold $M$ with $\eta=d f$ the $g$-dual of $\xi$. If $\eta$ is a harmonic form, then:

$$
\frac{1}{2} \Delta_{f}\left(|\xi|^{2}\right)=|\operatorname{Hess}(f)|^{2}+\lambda|\xi|^{2}+\mu|\xi|^{4}
$$

Using Corollary 2.4 we get: 
Corollary 3.1. Under the hypotheses of Theorem 3.1, if $M$ is of constant scalar curvature, then at least one of $\lambda$ and $\mu$ is non positive.

As a consequence for the case of gradient Ricci soliton, we have:

Proposition 3.2. Let $(g, \xi:=\operatorname{grad}(f), \lambda)$ be a gradient Ricci soliton on the $n$-dimensional manifold $M$ of constant scalar curvature, with $\eta=d f$ the $g$-dual of $\xi$. If $\eta$ is a harmonic form, then the soliton is shrinking.

Proof. From Theorem 2.4 and Theorem 3.1 we obtain $|H e s s(f)|^{2}+\lambda|\xi|^{2}=0$, hence $\lambda<0$.

Remark 3.3. i) Assume that $\mu \neq 0$. If $\lambda \geq-\mu|\xi|^{2}$, then $\Delta_{f}\left(|\xi|^{2}\right) \geq 0$ and from the maximum principle follows that $|\xi|^{2}$ is constant in a neighborhood of any local maximum. If $|\xi|$ achieve its maximum, then $M$ is quasiEinstein. Indeed, since Hess $(f)=0$, from the soliton equation we have $S=-\lambda g-\mu d f \otimes d f$. Moreover, in this case, $|\xi|^{2}\left(\lambda+\mu|\xi|^{2}\right)=0$, which implies either $\xi=0$ or $|\xi|^{2}=-\frac{\lambda}{\mu} \geq 0$. Since scal $+\lambda n+\mu|\xi|^{2}=0$ we get scal $=\lambda(1-n)$.

ii) For $\mu=0$, we get the Ricci soliton case [35].

Computing the gradient soliton equation in $\left(\gamma^{\sharp}, X\right), X \in \mathfrak{X}(M)$, we obtain:

$$
g\left(\nabla_{\gamma^{\sharp}} \xi, X\right)+g\left(Q \gamma^{\sharp}, X\right)+\lambda g\left(\gamma^{\sharp}, X\right)+\mu \eta\left(\gamma^{\sharp}\right) \eta(X)=0
$$

and taking $X:=\xi$ we get:

$$
\frac{1}{2} \gamma^{\sharp}\left(|\xi|^{2}\right)+\gamma(Q \xi)+\left(\lambda+\mu|\xi|^{2}\right) \eta\left(\gamma^{\sharp}\right)=0 .
$$

Therefore:

Proposition 3.3. Let $(g, \xi, \lambda, \mu)$ be an $\eta$-Ricci soliton on the $n$-dimensional manifold $M$ with $\eta$ the $g$-dual of $\xi$ and $\mu \neq 0$. If $\gamma$ is a 1-form, then $\gamma$ is orthogonal to $\eta$ if and only if

$$
\nabla_{\gamma^{\sharp}} \xi+Q \gamma^{\sharp}+\lambda \gamma^{\sharp}=0,
$$

hence:

$$
\frac{1}{2} \gamma^{\sharp}\left(|\xi|^{2}\right)=-\gamma(Q \xi) .
$$

Some results concerning the harmonic 1-forms on gradient $\eta$-Ricci solitons are further presented.

For two $(0,2)$-tensor fields $T_{1}$ and $T_{2}$, denote by $\left\langle T_{1}, T_{2}\right\rangle:=\sum_{1 \leq i, j \leq n} T_{1}\left(E_{i}, E_{j}\right) T_{2}\left(E_{i}, E_{j}\right)$, for $\left\{E_{i}\right\}_{1 \leq i \leq n}$ a local orthonormal frame field.

Theorem 3.2. Let $M$ be a compact and oriented $n$-dimensional manifold $M,(g, \xi:=\operatorname{grad}(f), \lambda, \mu)$ a gradient $\eta$-Ricci soliton with $\eta=d f$ the $g$-dual of $\xi$ and $\gamma$ a 1 -form.

1. If $\gamma$ is orthogonal to $\eta$ and $\mu \neq 0$, then $\gamma^{\sharp} \in \operatorname{ker}\left(\nabla_{\xi} \eta+\eta \circ Q\right)$.

2. If $\gamma$ is harmonic, then either we have a Ricci soliton or $\nabla_{\xi} \gamma^{\sharp} \in \operatorname{ker} \eta$.

3. If $\gamma$ is exact with $\gamma=d u$, then:

$$
\int_{M}\langle S, \operatorname{div}(d u)\rangle=-\int_{M}\langle\operatorname{Hess}(f), \operatorname{Hess}(u)\rangle-\mu\left(d f \mid \nabla_{\operatorname{grad}(f)} \operatorname{grad}(u)\right) .
$$

Moreover, if $\gamma$ is harmonic, the relation (3.12) becomes:

$$
\int_{M}\langle\operatorname{Hess}(f), \operatorname{Hess}(u)\rangle=-\mu\left(d f \mid \nabla_{\operatorname{grad}(f)} \operatorname{grad}(u)\right) .
$$

Proof. From (3.11) and using (3.1) we get:

$$
0=g\left(\nabla_{\gamma^{\sharp}} \xi, \xi\right)+g\left(Q \xi, \gamma^{\sharp}\right)=\xi\left(\eta\left(\gamma^{\sharp}\right)\right)-\eta\left(\nabla_{\xi} \gamma^{\sharp}\right)+g\left(\xi, Q \gamma^{\sharp}\right)=\left(\nabla_{\xi} \eta\right) \gamma^{\sharp}+\eta\left(Q \gamma^{\sharp}\right)
$$

and hence 1 . 
Let $\left\{E_{i}\right\}_{1 \leq i \leq n}$ be a local orthonormal frame field with $\nabla_{E_{i}} E_{j}=0$ in a point. For any symmetric $(0,2)$-tensor field $Z$ and any 1-form $\gamma$ :

$$
\begin{gathered}
\left\langle Z, L_{\gamma^{\sharp}} g\right\rangle=\sum_{1 \leq i, j \leq n} Z\left(E_{i}, E_{j}\right)\left(L_{\gamma^{\sharp}} g\right)\left(E_{i}, E_{j}\right)=2 \sum_{1 \leq i, j \leq n} Z\left(E_{i}, E_{j}\right) g\left(\nabla_{E_{i}} \gamma^{\sharp}, E_{j}\right)= \\
=2 \sum_{1 \leq i, j \leq n} Z\left(E_{i}, E_{j}\right) E_{i}\left(\gamma\left(E_{j}\right)\right)=2\langle Z, \operatorname{div}(\gamma)\rangle .
\end{gathered}
$$

Also:

$$
\left\langle g, L_{\gamma^{\sharp}} g\right\rangle=\sum_{i=1}^{n}\left(L_{\gamma^{\sharp}} g\right)\left(E_{i}, E_{i}\right)=2 \sum_{i=1}^{n} g\left(\nabla_{E_{i}} \gamma^{\sharp}, E_{i}\right)=2 \operatorname{div}\left(\gamma^{\sharp}\right)
$$

and

$$
\begin{gathered}
\left\langle d f \otimes d f, L_{\gamma^{\sharp}} g\right\rangle=\sum_{1 \leq i, j \leq n} d f\left(E_{i}\right) d f\left(E_{j}\right)\left(L_{\gamma^{\sharp}} g\right)\left(E_{i}, E_{j}\right)=2 \sum_{1 \leq i, j \leq n} d f\left(E_{i}\right) d f\left(E_{j}\right) g\left(\nabla_{E_{i}} \gamma^{\sharp}, E_{j}\right)= \\
=2 g\left(\nabla_{\operatorname{grad}(f)} \gamma^{\sharp}, \operatorname{grad}(f)\right)=2 g\left(\left(\nabla_{\operatorname{grad}(f)} \gamma\right)^{\sharp},(d f)^{\sharp}\right) .
\end{gathered}
$$

Computing $\langle S, \operatorname{div}(\gamma)\rangle$ by replacing $S$ from the $\eta$-Ricci soliton equation, we obtain:

$$
\langle S, \operatorname{div}(\gamma)\rangle=-\frac{1}{2}\left\langle\operatorname{Hess}(f), L_{\gamma^{\sharp}} g\right\rangle-\lambda \operatorname{div}\left(\gamma^{\sharp}\right)-\mu g\left(\left(\nabla_{\operatorname{grad}(f)} \gamma\right)^{\sharp},(d f)^{\sharp}\right) .
$$

For 2. we use $\operatorname{div}(\gamma)=0=\operatorname{div}\left(\gamma^{\sharp}\right)$ and for 3. we use the fact that $\gamma^{\sharp}=\operatorname{grad}(u)$, hence $L_{\gamma^{\sharp}} g=2 H \operatorname{ess}(u)$ and apply the divergence theorem.

Since

$$
\eta\left(\nabla_{\xi} \xi\right)=\frac{1}{2} \xi\left(|\xi|^{2}\right)
$$

and for $\eta$ harmonic:

$$
\int_{M}|H e s s(f)|^{2}=-\mu \int_{M} d f\left(\nabla_{\xi} \xi\right)
$$

we get:

Corollary 3.2. Under the hypotheses of Theorem 3.2, if $\eta$ is a harmonic form, then either we have a Ricci soliton or the potential vector field $\xi$ is of constant length. In the second case, $\eta$ is a solution of the Schrödinger-Ricci equation and $M$ is a quasi-Einstein manifold.

We know that the Bochner formula, for an arbitrary vector field $\xi$ [10], states:

$$
\frac{1}{2} \Delta\left(|\xi|^{2}\right)=|\nabla \xi|^{2}+S(\xi, \xi)+\xi(\operatorname{div}(\xi))
$$

and taking into account that the $g$-dual 1-form $\eta$ of $\xi$ satisfies

$$
|\xi|=|\eta|, \quad|\nabla \xi|=|\nabla \eta|, \quad S(\xi, \xi)=S^{\sharp}(\eta, \eta), \quad \xi(\operatorname{div}(\xi))=\langle\Delta(\eta), \eta\rangle,
$$

we have the corresponding relation for $\eta$ :

$$
\frac{1}{2} \Delta\left(|\eta|^{2}\right)=|\nabla \eta|^{2}+S^{\sharp}(\eta, \eta)+\langle\Delta(\eta), \eta\rangle .
$$

Let $\gamma$ be a 1-form and writing the previous relation for $\eta+\gamma$ we obtain:

$$
\frac{1}{2} \Delta(\langle\eta, \gamma\rangle)=\langle\nabla \eta, \nabla \gamma\rangle+S^{\sharp}(\eta, \gamma)+\frac{1}{2}(\langle\Delta(\eta), \gamma\rangle+\langle\Delta(\gamma), \eta\rangle) .
$$

Theorem 3.3. Let $M$ be an $n$-dimensional manifold, $(g, \xi:=\operatorname{grad}(f), \lambda, \mu)$ a gradient $\eta$-Ricci soliton with $\eta=d f$ the $g$-dual of $\xi$ and $\gamma$ a 1-form. Then:

$$
\frac{1}{2} \Delta(\langle d f, \gamma\rangle)=\langle H e s s(f), \nabla \gamma\rangle-\mu \Delta(f)\langle d f, \gamma\rangle+\frac{1}{2}\langle d f, \Delta(\gamma)\rangle .
$$


Proof. From (2.4), (3.3), (3.7) and $2 \operatorname{div}(S)=d($ scal), we get:

$$
S^{\sharp}(\eta, \gamma)=S\left(\xi, \gamma^{\sharp}\right)=-\frac{1}{2} d(\Delta(f))\left(\gamma^{\sharp}\right)-\mu \Delta(f) d f\left(\gamma^{\sharp}\right)=-\frac{1}{2}\langle\Delta(d f), \gamma\rangle-\mu \Delta(f)\langle d f, \gamma\rangle,
$$

hence (3.15).

Proposition 3.4. Let $M$ be an $n$-dimensional manifold, $(g, \xi:=\operatorname{grad}(f), \lambda, \mu)$ a gradient $\eta$-Ricci soliton with $\eta=d f$ the $g$-dual of $\xi$ and $\gamma$ a 1 -form.

1. If $\gamma$ is orthogonal to $\eta$, then $\langle\operatorname{Hess}(f), \nabla \gamma\rangle=-\frac{1}{2}\langle d f, \Delta(\gamma)\rangle$.

2. If $\gamma$ is harmonic, then $\frac{1}{2} \Delta(\langle d f, \gamma\rangle)=\langle\operatorname{Hess}(f), \nabla \gamma\rangle-\mu \Delta(f)\langle d f, \gamma\rangle$. In this case, $\langle d f, \gamma\rangle$ is harmonic if and only if $\mu \Delta(f)\langle d f, \gamma\rangle=\langle\operatorname{Hess}(f), \nabla \gamma\rangle$.

Moreover, if $\gamma$ is orthogonal to $\eta$, then $\nabla \gamma$ is orthogonal to $\nabla \eta$.

$L_{f}^{2}$ harmonic 1-forms. Endow the Riemannian manifold $(M, g)$ with the weighted volume form $e^{-f} d V$ and define $L_{f}^{2}$ forms those forms $\gamma$ satisfying $\int_{M}|\gamma|^{2} e^{-f} d V<\infty$.

The most natural operator of Laplacian-type associated to the weighted manifold $\left(M, g, e^{-f} d V\right)$ is the $f$ Laplace-Hodge operator

$$
\Delta_{f}:=\Delta-\nabla_{\operatorname{grad}(f)}
$$

which is self-adjoint with respect to this measure.

We say that a 1 -form $\gamma$ is $f$-harmonic if

$$
\Delta_{f}(\gamma)=0
$$

Remark that $\gamma$ is $f$-harmonic if and only if

$$
\Delta(\gamma)=i_{\nabla_{\gamma}^{\sharp} \xi} g .
$$

From (2.4) and (3.4) we deduce:

Proposition 3.5. Let $(g, \xi:=\operatorname{grad}(f), \lambda, \mu)$ be a gradient $\eta$-Ricci soliton on the $n$-dimensional manifold $M$ with $\eta=d f$ the $g$-dual of $\xi$. Then $\eta$ is an $f$-harmonic form if and only if scal $+\left(\mu+\frac{1}{2}\right)|\xi|^{2}$ is constant.

In terms of $\Delta_{f}$, the relation (3.14) can be written [34]:

$$
\frac{1}{2} \Delta_{f}\left(|\gamma|^{2}\right)=|\nabla \gamma|^{2}+S_{f}^{\sharp}(\gamma, \gamma)+\left\langle\Delta_{f}(\gamma), \gamma\right\rangle,
$$

where $S_{f}:=\operatorname{Hess}(f)+S$ is the Bakry-Émery Ricci tensor.

Using a Reilly-type formula involving the $f$-Laplacian, an interesting result was obtained in [29], namely, if the manifold $M$ is the boundary of a compact and connected Riemannian manifold and has non negative $m$-dimensional Bakry-Émery Ricci curvature and non negative $f$-mean curvature, then either $M$ is connected or it has only two connected components, in the later case, being totally geodesic.

Another interesting topological property will be stated in the next theorem:

Theorem 3.4. Let $\left(M^{n}, g, e^{-f} d V\right)$ be a complete, non compact smooth metric measure space and $(g, \xi:=\operatorname{grad}(f), \lambda, \mu)$ a gradient $\eta$-Ricci soliton with $\eta=d f$ the $g$-dual of $\xi$. If there exists a non trivial $L_{f}^{2}$ harmonic 1 -form $\gamma_{0}$ such that $\lambda\left|\gamma_{0}\right|^{2}+\mu\left(\gamma_{0}(\xi)\right)^{2} \leq 0$, then $M$ has finite volume and its universal covering splits isometrically into $\mathbb{R} \times N^{n-1}$.

Proof. The condition $\lambda\left|\gamma_{0}\right|^{2}+\mu\left(\gamma_{0}(\xi)\right)^{2} \leq 0$ is equivalent to $S_{f}^{\sharp}\left(\gamma_{0}, \gamma_{0}\right) \geq 0$. From (3.16) and Lemma 3.2 from [38]:

$$
\left|\gamma_{0}\right| \Delta_{f}\left(\left|\gamma_{0}\right|\right) \geq 0
$$

Following the same steps as in [38], we obtain the conclusion.

Remark 3.4. i) Under the hypothesis of Theorem 3.4, in particular, we deduce that $\gamma_{0}$ is $\nabla$-parallel and of constant length. Also, $\lambda \leq 0$ since in [36] was shown that $\lambda>0$ implies $M$ compact.

ii) In the Ricci soliton case, the hypothesis of Theorem 3.4 requires that the space of $L_{f}^{2}$ harmonic 1 -forms to be nonempty and the Ricci soliton to be shrinking in order to get the same conclusion. 


\section{References}

[1] Blaga, A. M.: A note on almost $\eta$-Ricci solitons in Euclidean hypersurfaces. Serdica Math. J. 43 (3-4), 361-368 (2017).

[2] Blaga, A. M.: A note on warped product almost quasi-Yamabe solitons. Filomat 33 (7), 2009-2016 (2019).

[3] Blaga, A. M.: Almost $\eta$-Ricci solitons in $(L C S)_{n}$-manifolds. Bull. Belg. Math. Soc. Simon Stevin 25 (5), 641-653 (2018).

[4] Blaga, A. M.: $\eta$-Ricci solitons on Lorentzian para-Sasakian manifolds. Filomat 30 (2), 489-496 (2016).

[5] Blaga, A. M.: $\eta$-Ricci solitons on para-Kenmotsu manifolds. Balkan J. Geom. Appl. 20 (1), 1-13 (2015)

[6] Blaga, A. M.: Last multipliers on $\eta$-Ricci solitons. Matematichki Bilten 42 (2), $85-90$ (2018).

[7] Blaga, A. M.: On gradient $\eta$-Einstein solitons. Kragujevak J. Math. 42 (2), 229-237 (2018).

[8] Blaga, A. M.: On harmonicity and Miao-Tam critical metrics in a perfect fluid spacetime. To appear in Bol. Soc. Mat. Mexicana.

[9] Blaga, A. M.: On solitons in statistical geometry. Int. J. Appl. Math. Stat. 58 (4), (2019).

[10] Blaga, A. M.: On warped product gradient $\eta$-Ricci solitons. Filomat 31 (18), 5791-5801 (2017).

[11] Blaga, A. M.: Remarks on almost $\eta$-solitons. Matematicki Vesnik 71 (3), 244-249 (2019).

[12] Blaga, A. M.: Solitons and geometrical structures in a perfect fluid spacetime. To appear in Rocky Mountain J. Math.

[13] Blaga, A. M.: Solutions of some types of soliton equations in $\mathbb{R}^{3}$. Filomat 33 (4), 1159-1162 (2019).

[14] Blaga, A. M.: Some geometrical aspects of Einstein, Ricci and Yamabe solitons. J. Geom. Sym. Phys. 52, 17-26 (2019).

[15] Blaga, A. M., Baishya, K. K., Sarkar, N.: Ricci solitons in a generalized weakly (Ricci) symmetric D-homothetically deformed Kenmotsu manifold. Ann. Univ. Paedagog. Crac. Stud. Math. 18, 123-136 (2019).

[16] Blaga, A. M., Crasmareanu, M. C.: Inequalities for gradient Einstein and Ricci solitons. To appear in Facta Univ. Math. Inform.

[17] Blaga, A. M., Crasmareanu, M. C.: Torse-forming $\eta$-Ricci solitons in almost paracontact $\eta$-Einstein geometry. Filomat 31 (2), $499-504$ (2017).

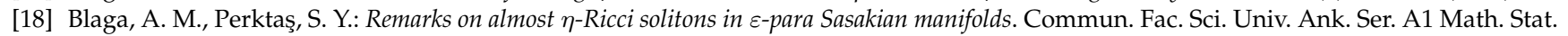
68 (2), 1621-1628 (2019).

[19] Blaga, A. M., Perktaş, S. Y., Acet, B. E., Erdogan, F. E.: $\eta$-Ricci solitons in $\varepsilon$-almost paracontact metric manifolds. Glasnik Matematicki 53 (1), 377-410 (2018).

[20] Catino, G.: A note on four-dimensional (anti-)self-dual quasi-Einstein manifolds. Differential Geom. Appl. 30, 660-664 (2012).

[21] Călin, C., Crasmareanu, M.: Eta-Ricci solitons on Hopf hypersurfaces in complex space forms. Rev. Roumaine Math. Pures Appl. 57 (1), $55-63$ (2012).

[22] Cho, J. T., Kimura, M.: Ricci solitons and real hypersurfaces in a complex space form. Tohoku Math. J. 61 (2), $205-212$ (2009).

[23] Chaki, M. C., Maity, R. K.: On quasi Einstein manifolds. Publ. Math. Debrecen 57, 297-306 (2000).

[24] Chow, B., Chu, S.-C., Glickenstein, D., Guenther, C., Isenberg, J., Ivey, T., Knopf, D., Lu, P., Luo, F., Ni, L.: The Ricci Flow: Techniques and Applications: Part I: Geometric Aspects 135. AMS (2007).

[25] Chow, B., Lu, P., Ni, L.: Hamilton's Ricci Flow. Graduate Studies in Mathematics 77. AMS (2006).

[26] De, K., Blaga, A. M., De, U. C.: *-Ricci solitons on ( $\varepsilon$ )-Kenmotsu manifolds. To appear in Palestine Math. J.

[27] De, U. C., Ghosh, G. C.: On quasi-Einstein and special quasi-Einstein manifolds, Proc. of the Int. Conf. of Mathematics and its Applications, Kuwait University, April 5-7, 2004, 178-191.

[28] De, U. C., Ghosh, G. C.: On quasi-Einstein manifolds. Period. Math. Hungar. 48 (1-2), 223-231 (2004).

[29] Deng, H.: Compact manifolds with positive m-Bakry-Émery Ricci tensor. Differential Geom. Appl. 32, 88-96 (2014).

[30] Deszcz, R., Hotlos, M., Senturk, Z.: On curvature properties of quasi-Einstein hypersurfaces in semi-Euclidean spaces. Soochow J. Math. 27, 375-389 (2001).

[31] Hamilton, R. S.: The Ricci flow on surfaces, in Mathematics and General Relativity, Contemp. Math. 71. AMS, 237-262 (1988).

[32] Hamilton, R. S.: Three-manifolds with positive Ricci curvature. J. Differential Geom. 17 (2), 255-306 (1982).

[33] Lima, E. L.: Grupo Fundamental e Espaços de Recobrimento. IMPA (2012).

[34] Lot, J.: Some geometric properties of the Bakry-Émery Ricci tensor. Comment. Math. Helv. 78 (4), 865-883 (2003).

[35] Petersen, P., Wylie, W.: Rigidity of gradient Ricci solitons. Pacific J. Math. 241 (2), 329-345 (2009).

[36] Qian, Z.: Estimates for weighted volumes and applications. Quart. J. Math. Oxford Ser. (2) 48 (190), 235-242 (1997).

[37] Smale, S.: On gradient dynamical systems. Ann. of Math. 2 (74), 199-206 (1961).

[38] Vieira, M.: Harmonic forms on manifolds with non-negative Bakry-Émery Ricci curvature. Archiv der Mathematik 101 (6), $581-590$ (2013).

\section{Affiliation}

ADARA M. BLAGA

AdDress: West University of Timişoara, Dept. of Mathematics, Bld. V. Pârvan nr. 4, 300223, TimişoaraRomânia.

E-MAIL: adarablaga@yahoo.com

ORCID ID: 0000-0003-0237-3866 